\title{
Free Vibration Response of a Multilayer Smart Hybrid Composite Plate with Embedded SMA Wires
}

\begin{abstract}
In this paper, free vibration response of a hybrid composite plate was studied. Effects of some geometrical, physical and material parameters on response of the composite plates embedded with shape memory alloy (SMA) wires were investigated, which have not been reported in the literature thus far. Some of these parameters included important factors affecting free vibration response of the smart hybrid composite plates. The SMA wires were embedded within the layers of the composite laminate. First-order shear deformation theory (FSDT) was utilized to obtain the governing equations of hybrid composite plates. Transverse shear and rotary inertia effects of the plate were taken into consideration. For simply-supported boundary conditions, systematic closed form solutions were obtained by Navier's technique. It was established that dynamic behavior of the smart hybrid composite plate depended on various parameters such as volume fraction, temperature dependent recovery stress and tensile pre-strain of SMA wires and aspect ratio of the laminated hybrid plate.
\end{abstract}

K. Malekzadeh ${ }^{\mathrm{a}}$,

A. Mozafari ${ }^{b}$ and

Faramarz Ashenai Ghasemi ${ }^{*}$ c

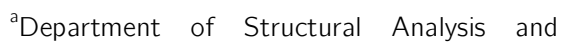
Simulation, Space research institute,

Tehran-Karaj Highway, Post box: 13445-768, Tehran, IRAN.

${ }^{\mathrm{b}}$ Department of Mechanical Engineering, $\mathrm{K}$. N. Toosi University of Technology, Vafadar East Ave, 4th Tehran pars Sq., Tehran, Iran.

${ }^{\mathrm{c} D e p a r t m e n t}$ of Mechanical Engineering, Shahid Rajaee Teacher Training University (SRTTU), Lavizan, Postal Code 1678815811,Tehran, Iran.

Received in 28 Feb 2013

In revised form 10 Jun 2013

*Author email: F.a.ghasemi@srttu.edu

\section{Keywords}

Free vibrations, Smart hybrid composite, SMA wires, Recovery stress.

\section{INTRODUCTION}

Shape memory alloys (SMAs) are characterized by their large internal forces, unique ability of changing their material properties, wide range of operational temperature, excellent damping properties and high durability. Using shape memory alloys as fiber reinforcement gives numerous adaptive capabilities to structures (Zhang et al., 2006 and $\mathrm{Ni}$ et al., 2007), one of which is controlling motion and vibration of structures. 


\section{NOTATIONS}

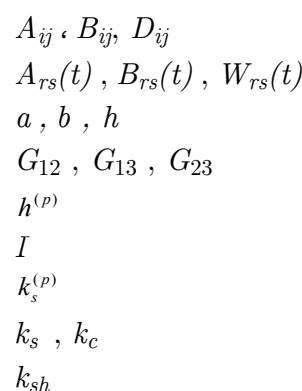

[K]

[M]

$N, M$

$N^{i}, N^{i}$

$N^{r}, M^{r}$

$N^{T}, M^{T}$

$Q_{i j}$

$Q_{i j}^{m}$

$\left[Q^{(p)}{ }_{i j}\right]$

$q$

$\left(\bar{Q}_{i j}^{(p)}\right)$

$u^{0}, v^{0}, w^{0}$

$\Delta T$

[T]

$\alpha^{c(p)}$

$a_{i}^{c}(i=l, t)$

$\beta^{(p)}$

$\theta^{(p)}$

$\gamma_{12}, \gamma_{13}, \gamma_{32}$

$\gamma_{x y}, \gamma_{y z}, \gamma_{z x}$

$\stackrel{\circ}{\boldsymbol{\varepsilon}}, x$

$\varepsilon_{1} \cdot \varepsilon_{2}$

$\varepsilon_{x}, \varepsilon_{y}$

$\varepsilon_{x z}, \varepsilon_{y z}$

$v_{i j}$

$\varrho$

$\rho_{0}{ }^{(p)}$

$\sigma_{1}$ ، $\sigma_{2}$

$\sigma_{x z} ، \sigma_{y z}$

$\sigma_{r}$

$\tau_{12}, \tau_{13}, \tau_{23}$

$\psi_{x}, \psi_{y}$

$\omega_{r s}$ matrices of extensional, coupling and bending stiffness

time dependent coefficients for Fourier transform

length, width and thickness of the plate

shear modulus in principal directions of composite plate

composite lamina thickness of the pth lamina

inertia moment of plate

volume fraction of SMA wires of the pth lamina

volume fraction of SMA wires and composite medium

shear correction factor

symmetric stiffness matrix

symmetric mass matrix

tensile force and moment per unit length

tensile force per unit length in $\mathrm{x}$ and $\mathrm{y}$ direction

tensile recovery force and recovery moment per unit length

tensile thermal force and thermal moment per unit length

reduced stiffness matrices of the SMA hybrid composite

reduced stiffness matrices of composite medium

reduced stiffness matrix of the lamina in the principal material coordinates

load function

transformed reduced stiffness components

plate displacements in $\mathrm{x}, \mathrm{y}$ and $\mathrm{z}$ directions at plate mid-plane

temperature difference between current and reference temperatures.

transformation matrix

coefficient of thermal expansion of composite medium in the pth lamina

longitudinal and transverse thermal expansion coefficients of composite medium

angle of SMA wires of the pth lamina

angle of fiber of the pth lamina

principal shear strains of plate

shear strains of plate in xy, yz and zx planes

midplane strains and curvatures

principal normal strains of the plate

principal normal strains of the plate in the $\mathrm{x}$, and $\mathrm{y}$ directions

shear strains of plate in $\mathrm{xz}, \mathrm{yz}$ planes

Poisson's ratio in principal directions of composite plate

density of the whole plate

density of the pth lamina of the plate

principal normal stresses of plate

shear stresses of plate in $\mathrm{xz}$, yz planes

tensile recovery stress

principal shear stresses of plate

shear rotations in the $\mathrm{x}$ and $\mathrm{y}$ directions

fundamental frequencies of the plate 
A limited number of papers concerning natural vibration of SMA fiber-reinforced composite plates can be found in the present literature. Zhong et al. (2006) proposed an experimental test procedure in order to measure fundamental natural frequency of smart plates. Two types of laminated composite plates with unidirectional and woven SMAs were fabricated and their vibration characteristics were investigated by both impact vibration tests and theoretical analysis. Stiffness and vibration characteristics of SMA/ER3 composites with shape memory alloy short fibers were experimentally investigated by $\mathrm{Ni}$ et al. (2007) experimentally. Lau (2002) investigated vibration characteristic of SMA composite beam in different boundary conditions using finite element method (FEM) and showed that increasing temperature in the composite beam with pre-strained SMA fibers caused increase of natural frequency and damping ratios of smart composite beams. Vibration of thermally post-buckled composite plates embedded with shape memory alloy fibers was studied by Park et al. (2004) using FEM and first shear deformation theory.

Numerical results representing critical temperature increased and thermal large deflection decreased using SMA fibers. Ostachowicz and Kaczmarczyk (2001) analyzed the dynamic response of composite laminated plate with a delamination defect subjected to aerodynamic loads using FEM. The results demonstrated that flutter boundaries can be modified using SMA fibers embedded in the plate. Ostachowicz et al. (1999) studied the influence of SMA fibers upon natural frequencies of the plate using FEM. The results of numerical calculations showed that SMA fibers can greatly influence natural frequencies of the plate. Ostachowicz et al. (2000) illustrated influence of SMA fibers upon changes in natural frequencies and thermal buckling of a composite multilayer plate with SMA components.

Birman et al. (1996) demonstrated that, if SMA wires were embedded in traditional polymer composites, tensile stresses could be generated inside the structures. Hence, impact resistance of the structures would be increased. He also demonstrated that, if some SMA wires were embedded in different layers of the structure, the global deflection of the structure would be reduced in low velocity impacts. Khalili et al. (2007) also studied effect of smart stiffening procedure on low-velocity impact response of smart structures.

In the present research, free vibration response of a rectangular smart hybrid composite plate was studied. The effect of some geometrical, physical and material parameters on the response of the composite plate embedded with SMA wires was also investigated. Some of the numerical results (which have not been reported in the literature so far) are presented in this paper.

\section{CONSTITITIVE EQUATIONS}

Constitutive equations of principal stress-strain relationship for a SMA hybrid laminated composite are as follows (Ostachowicz et al., 2000, Birman et al. 1996 and Khalili et al., 2007): 


$$
\begin{gathered}
\left\{\begin{array}{c}
\sigma_{1} \\
\sigma_{2} \\
\tau_{12}
\end{array}\right\}=\left\{\begin{array}{ccc}
Q_{11} & Q_{12} & 0 \\
Q_{12} & Q_{22} & 0 \\
0 & 0 & Q_{66}
\end{array}\right\}\left\{\begin{array}{c}
\varepsilon_{1} \\
\varepsilon_{2} \\
\gamma_{12}
\end{array}\right\}+\left\{\begin{array}{c}
\sigma_{r} \\
0 \\
0
\end{array}\right\} k_{\mathrm{s}}\left[\begin{array}{ccc}
Q_{11}^{m} & Q_{12}^{m} & 0 \\
Q_{12}^{m} & Q_{22}^{m} & 0 \\
0 & 0 & Q_{66}^{m}
\end{array}\right]\left\{\begin{array}{c}
\alpha_{l}^{c} \\
\alpha_{t}^{c} \\
0
\end{array}\right\} k_{c} \Delta T \\
\left\{\begin{array}{l}
\tau_{13} \\
\tau_{23}
\end{array}\right\}=\left\{\begin{array}{ll}
Q_{55} 0 \\
0 & Q_{44}
\end{array}\right\}\left\{\begin{array}{l}
\gamma_{13} \\
\gamma_{23}
\end{array}\right\}
\end{gathered}
$$

In the above equation, $\{\sigma\}$ and $\{\varepsilon\}$ represent stresses and strains in the principle directions, respectively. $Q_{i j}$ and $Q_{i j}^{m}$ are reduced stiffness matrices for the SMA hybrid composite and composite medium (without the SMA wires), respectively. or represents the recovery stress which can be determined analytically or experimentally (Birman et al. 1996 and Khalili et al., 2007). $a^{c}{ }_{i}(i=l, t)$ shows thermal expansion coefficients of the composite medium, calculated based on the temperature difference $\Delta T$ between the current and reference temperatures. Because of discontinuity function of stresses through the thickness between the layers, it is possible to determine constitutive equations considering the force-couple resultants in terms of stresses using integration of Equation (1) through the plate thickness, which yields:

$$
\begin{gathered}
\left\{\begin{array}{c}
N \\
M
\end{array}\right\}=\left[\begin{array}{ll}
\mathrm{A}_{\mathrm{ij}} & \mathrm{B}_{\mathrm{ij}} \\
\mathrm{B}_{\mathrm{ij}} & \mathrm{D}_{\mathrm{ij}}
\end{array}\right]\left\{\begin{array}{c}
\varepsilon^{0} \\
\kappa
\end{array}\right\}+\left\{\begin{array}{c}
\mathrm{N}^{\mathrm{i}} \\
\mathrm{M}^{\mathrm{i}}
\end{array}\right\} ;\left\{\begin{array}{c}
N^{i} \\
M^{i}
\end{array}\right\}=\left\{\begin{array}{c}
\mathrm{N}^{\mathrm{r}}-N^{T} \\
\mathrm{M}^{\mathrm{r}}-M^{T}
\end{array}\right\} ; i, j=1,2,6 \\
\{S\}=\left[k_{s h} A_{i j}\right]\{\gamma\} ; \quad i, j=4,5
\end{gathered}
$$

where $N$ and $S$ are vectors of forces and $M$ is vector of moments, respectively. $N^{r}$ and $M^{r}$ are vectors of recovery stress resultants and the stress moments generated in the SMA wires, respectively. $N^{T}$ and $M^{T}$ are vectors of the thermal stress resultants and the moments in the structure, respectively. Also, $\varepsilon^{0}$ and $\gamma$ are the mid-plane and shear strains and $x$ is the curvatures. In addition, ksh is the shear correction factor (Khalili et al., 2005, Whitney and Pagano, 1970 and Mindlin, 1951). In symmetrically laminated cross-ply plates:

$$
B_{i j}=M_{i}=M_{r}=M_{T}=0
$$

Also, in Equation (2) (Khalili et al., 2007):

$$
\begin{gathered}
N^{i}=\left\{\begin{array}{c}
N_{x}^{i} \\
N_{y}^{i} \\
N_{x y}^{i}
\end{array}\right\}=\left\{\begin{array}{c}
N_{x}^{r}-N_{x}^{T} \\
N_{y}^{r}-N_{y}^{T} \\
N_{x y}^{r}-N_{x y}^{T}
\end{array}\right\} \\
\left\{\begin{array}{c}
N_{x}^{r} \\
N_{y}^{r} \\
N_{x y}^{r}
\end{array}\right\}=\sum_{p=1}^{n} \sigma_{r}\left\{\begin{array}{c}
k_{s}^{(p)} h^{(p)} \cos ^{2}\left(\beta^{(p)}\right) \\
k_{s}^{(p)} h^{(p)} \sin ^{2}\left(\beta^{(p)}\right) \\
2 k_{s}^{(p)} h^{(p)} \sin \left(\beta^{(p)}\right) \cos \left(\beta^{(p)}\right)
\end{array}\right\}
\end{gathered}
$$

Latin American Journal of Solids and Structures 11(2014) $279-298$ 


$$
\left\{\begin{array}{c}
N_{x}{ }^{T} \\
N_{y}{ }^{T} \\
N_{x y}{ }^{T}
\end{array}\right\}=\Delta T \sum_{p=1}^{n} \bar{Q}^{(p)} \alpha^{c(p)} h^{(p)} k_{c}^{(p)}
$$

is the transformed reduced stiffness matrix of the $p^{\text {th }}$ layer, defined as: $\left[\bar{Q}_{i j}^{(p)}\right]$ where:

$$
\begin{array}{cc}
{\left[\bar{Q}_{i j}^{(p)}\right]=\left[T_{1}\right]^{-1}\left[Q^{(p)}{ }_{i j}\right]\left[T_{1}\right]^{-T}} & (i, j=1,2,6, r=t, b) \\
{\left[\bar{Q}_{i j}^{(p)}\right]=\left[T_{2}\right]^{-1}\left[Q^{(p)}{ }_{i j}\right]\left[T_{2}\right]^{-T}} & (i, j=5,4)
\end{array}
$$

in which $\left[T_{1}\right]_{K}$ and $\left[T_{2}\right]_{K}$ are transformation matrices for the $k^{t h}$ layer and $\left[Q^{(p)}{ }_{i j}\right]$ is the reduced stiffness matrix of the lamina in the principal material coordinates, as given in Reddy (1997). In this paper, in all of the SMA embedded composite laminas, fibers of composite lamina and SMA wires can be in different directions. $\sigma_{r}$ is recovery stress and a function of temperature and prestrain of the SMA wires.

Figure 1 (see Ostachowicz et al., 1999 and Ostachowicz et al., 2000) is used in a tabulated form in the computation and dynamic analyses. In Equation (5), $\alpha^{c(p)}$ and $k_{c}^{(p)}$ are coefficients of thermal expansion and volume fraction of composite medium in the pth ply, calculated in the following equations (Reddy, 1997 and Altenbach et al., 2004):

$$
\alpha^{c(p)}=\left\{\begin{array}{l}
\alpha_{11}^{c(p)} \\
\alpha_{22}^{c(p)} \\
\alpha_{12}^{c(p)}
\end{array}\right\}=\left\{\begin{array}{c}
\alpha_{l}^{c(p)} \cos ^{2}\left(\theta^{(p)}\right)+\alpha_{t}^{c(p)} \sin ^{2}\left(\theta^{(p)}\right) \\
\alpha_{l}^{c(p)} \sin ^{2}\left(\theta^{(p)}\right)+\alpha_{t}^{c(p)} \cos ^{2}\left(\theta^{(p)}\right) \\
\left(\alpha_{l}^{c(p)}-\alpha_{t}^{c(p)}\right) \sin \left(\theta^{(p)}\right) \cos \left(\theta^{(p)}\right)
\end{array}\right\}, \quad k_{c}^{(p)}=1-k_{s}^{(p)}
$$

In plies with no SMA fibers, $V_{c}^{(k)}=1$. In Equation $(7), \theta^{(p)}$ is the angle of fiber of the $p^{\text {th }}$ lamina without embedded SMA wires. 


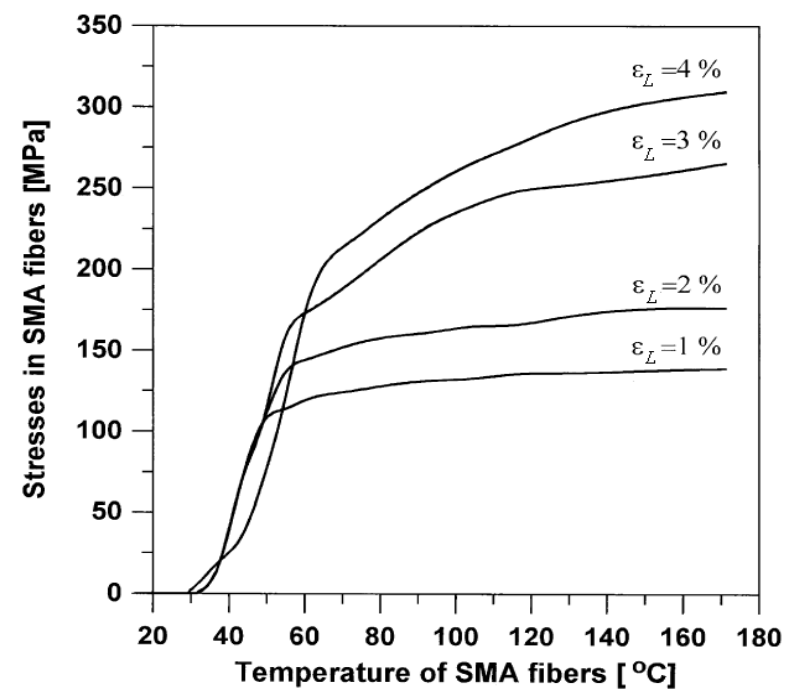

Figure 1 Recovery stress-temperature curves of the SMA wires in different pre-strain values, $\mathcal{E}_{L}$ (Ostachowicz et al., 1999 and Ostachowicz and Krawczuk 2000).

\section{GOVERNING EQUATIONS AND NAVIER'S SOLUTION}

The plate equations developed by Whitney and Pagano (1970) were used since they included effect of transverse shear deformations (Figure 2). The assumed displacement field was:

$$
\begin{aligned}
& u=u^{0}(x, y, t)+z \psi_{x}(x, y, t) \\
& v=v^{0}(x, y, t)+z \psi_{y}(x, y, t) \\
& w=w^{0}(x, y, t)
\end{aligned}
$$

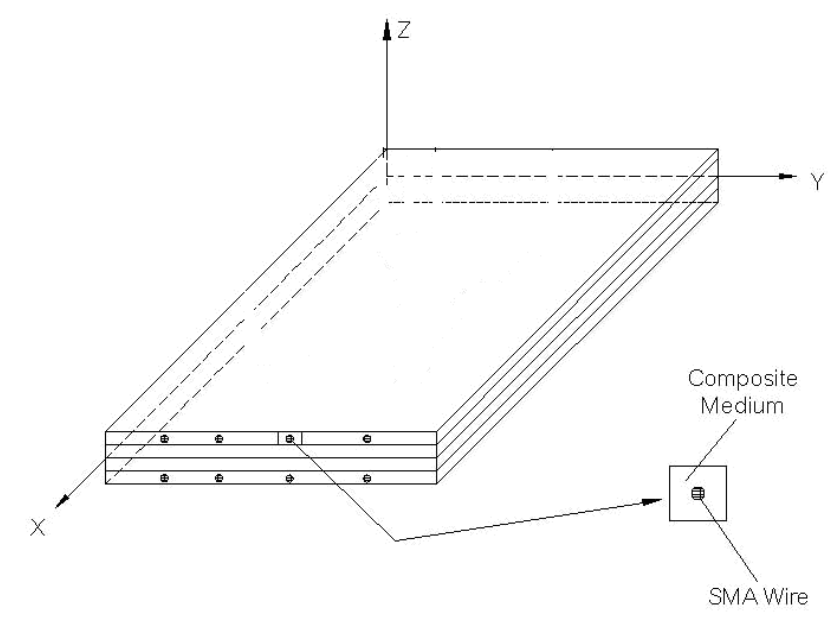

Figure 2 Schematic view of the SMA hybrid composite plate (Khalili et al., 2007). 
Reducing the equation to a specially symmetric, cross ply orthotropic form $\left(B_{i j}=0, A_{16}=A_{26}\right.$ $=D_{16}=D_{26}=0$ ) and adding the uniform in-plane initial stress resultants $\mathrm{N}_{\mathrm{x}}^{\mathrm{i}}$ and $\mathrm{N}_{\mathrm{y}}^{\mathrm{i}}$ (see Equations (4-5)) resulted in (Mindlin, 1951 and Reddy, 1997):

$$
\begin{gathered}
D_{11} \psi_{x},_{x x}+D_{66} \psi_{x},_{y y}+\left(D_{12}+D_{66}\right) \psi_{y},_{x y}-k_{s h} A_{55} \psi_{x}-k_{s h} A_{55} w,_{x}=I \ddot{\psi} \ddot{x}_{x} \\
\left(D_{12}+\mathrm{D}_{66}\right) \psi_{x},_{x y}+D_{66} \psi_{y},_{x x}+D_{22} \psi_{y},_{y y}-k_{s h} A_{44} \psi_{y}-k_{s h} A_{44} w,_{y}=I \ddot{\psi} \\
k_{s h} \mathrm{~A}_{55} \psi_{x},_{x}+\left(k_{s h} A_{55}+\mathrm{N}_{\mathrm{x}}^{\mathrm{i}}\right) \mathrm{w},_{x x}+k_{s h} A_{44} \psi_{y},_{y}+\left(k_{s h} A_{44}+\mathrm{N}_{\mathrm{y}}^{\mathrm{i}}\right) w,_{y y}+q=\rho \ddot{w}
\end{gathered}
$$

$k_{s h}$ is the shear correction factor introduced by Mindlin (1951), normally taken to be $\pi^{2} / 12$. In addition, $q$ is the dynamic normal load (transverse impact) over the plate and:

$$
\begin{gathered}
\left(A_{i j}, B_{i j}, D_{i j}\right)=\int_{-h / 2}^{h / 2}\left[\bar{Q}_{i j}^{(p)}\right]\left(1, z, z^{2}\right) d z \\
(I)=\int_{-h / 2}^{h / 2} \rho\left(z^{2}\right) \mathrm{d} z=\frac{1}{3} \sum_{k=1}^{n} \rho_{0}^{(p)}\left(\left(h^{(p)}\right)^{3}-\left(h^{(p-1)}\right)^{3}\right) \\
\rho=\left(\sum_{p=1}^{n} \rho_{0}{ }^{(p)} h^{(p)}\right)
\end{gathered}
$$

$\left(\bar{Q}_{i j}^{(p)}\right)(i, j=1,2,6)$ are transformed reduced in-plane stiffness components and $\left(\bar{Q}_{i j}^{(p)}\right)(i, j=4,5)$ are the transformed reduced transverse shear stiffness components, defined in Reddy (1997) and Altenbach et al. (2004).

In this work, attention was focused upon a simply supported rectangular plate with dimensions of a and b. Hence, the boundary conditions were as follows:

$$
\begin{aligned}
& w=\psi_{x},{ }_{x}=\psi_{y}=0 \quad ; \quad \text { at } \mathrm{x}=0, \mathrm{a} \\
& w=\psi_{y},{ }_{y}=\psi_{x}=0 \quad ; \quad \text { at } \mathrm{y}=0, \mathrm{~b}
\end{aligned}
$$

Figure 2 shows a SMA hybrid composite plate Khalili et al. (2007). The free vibration solution for a rectangular plate with simply supported boundary conditions at the upper and the lower face sheets was assumed to be of the following form:

$$
\begin{aligned}
w(x, y) & =\sum_{r, s=1}^{\infty} W_{r s} \sin \left(\alpha_{r} x\right) \sin \left(\beta_{s} y\right) \exp \left(i \omega_{r s} t\right), \quad \alpha_{r}=\frac{r \pi}{a} \\
\psi_{x}(x, y) & =\sum_{r, s=1}^{\infty} A_{r s} \cos \left(\alpha_{r} x\right) \sin \left(\beta_{s} y\right) \exp \left(i \omega_{r s} t\right), \quad \beta_{s}=\frac{s \pi}{b} \\
\psi_{y}(x, y) & =\sum_{r, s=1}^{\infty} B_{r s} \sin \left(\alpha_{r} x\right) \cos \left(\beta_{s} y\right) \exp \left(i \omega_{r s} t\right)
\end{aligned}
$$


The above double Fourier series of Dirichlet type functions can satisfy boundary conditions of the plate. In Equation (12), $W_{r s}, A_{r s}$ and $B_{r s}$ are unknown Fourier coefficients, $\mathrm{r}$ and s are half wave numbers in $\mathrm{x}$ and y directions, respectively, and $\omega$ is the vibration angular frequency. Hence, closed form solutions of the governing equations for free vibration of simply-supported rectangular composite plates with cross ply face sheets were obtained by applying Navier's technique. A $3 \times 3$ array of eigenvalue equations was also obtained. Since each coefficient of the Fourier series was independent, a one-term solution existed. Thus, the eigenvalue problem (Wilkinson, 1992) yielded only 3 eigenfrequencies for specified values of $r$ and $s$. Then, Equation (9) could be reduced to the following eigenvalue equation:

$$
\left(\left[\begin{array}{lll}
K_{11} & K_{12} & K_{13} \\
K_{21} & K_{22} & K_{23} \\
K_{31} & K_{32} & K_{33}
\end{array}\right]-\left[\begin{array}{ccc}
I & 0 & 0 \\
0 & I & 0 \\
0 & 0 & \rho
\end{array}\right] \omega_{r s}^{2}\right)\left\{\begin{array}{c}
A_{r s} \\
B_{r s} \\
W_{r s}
\end{array}\right\}=0
$$

where:

$$
\begin{aligned}
& K_{11}=D_{11}\left(\frac{r \pi}{a}\right)^{2}+D_{66}\left(\frac{s \pi}{b}\right)^{2}+k_{s h} A_{55} \\
& K_{12}=K_{21}=\left(D_{12}+D_{66}\right)\left(\frac{r \pi}{a}\right)\left(\frac{s \pi}{b}\right) \\
& K_{13}=K_{31}=k_{s h} A_{55}\left(\frac{r \pi}{a}\right) \\
& K_{22}=D_{66}\left(\frac{r \pi}{a}\right)^{2}+D_{22}\left(\frac{s \pi}{b}\right)^{2}+k_{s h} A_{44} \\
& K_{23}=K_{32}=k_{s h} A_{44}\left(\frac{s \pi}{b}\right) \\
& K_{33}=\left(k_{s h} A_{55}+N_{x}^{i}\right)\left(\frac{r \pi}{a}\right)^{2}+\left(k_{s h} A_{44}+N_{y}^{i}\right)\left(\frac{s \pi}{b}\right)^{2}
\end{aligned}
$$

Equation (13) can be written as:

$$
\left([K]-[M] \omega_{r s}^{2}\right)\{X\}=0
$$

where:

$$
\{X\}=\left\{\begin{array}{c}
A_{r s} \\
B_{r s} \\
W_{r s}
\end{array}\right\}
$$


The eigenvalue problem of Equation (15) is known as the standard eigenvalue problem (Khalili et al., 2005 and Reddy, 1997). By solving Equation (16), the eigenvalues can be analytically obtained (Khalili et al., 2007, Khalili et al., 2005 and Reddy, 1992):

$$
\operatorname{det}\left([K]-[M] \omega_{r s}^{2}\right)=0
$$

Then, eigenvectors $\{\mathrm{X}\}$ can be analytically calculated using Equation (15). The shape modes of the plate can be calculated using Equation (12). In addition, using the following equations as discussed earlier (Khalili et al., 2005), values of strains $\left(\boldsymbol{\varepsilon}_{x}, \boldsymbol{\varepsilon}_{y}, \boldsymbol{\gamma}_{x y}, \boldsymbol{\gamma}_{x z}\right.$ and $\left.\boldsymbol{\gamma}_{y z}\right)$ would be easily calculated:

$$
\begin{gathered}
\varepsilon_{x}=z \cdot \psi_{x, x}=-z \sum_{r, s=1}^{\infty} A_{r s} \cdot\left(\frac{\mathrm{r} \pi}{\mathrm{a}}\right) \cdot \operatorname{Sin}\left(\frac{r \pi}{a}\right) x \cdot \operatorname{Sin}\left(\frac{s \pi}{b}\right) y \\
\varepsilon_{y}=z \cdot \psi_{y, y}=-z \sum_{r, s=1}^{\infty} B_{r s} \cdot\left(\frac{\mathrm{s} \pi}{\mathrm{b}}\right) \cdot \operatorname{Sin}\left(\frac{r \pi}{a}\right) x \cdot \operatorname{Sin}\left(\frac{r \pi}{b}\right) y \\
\gamma_{x y}=z\left(\psi_{x, y}+\psi_{y, x}\right)=-z \sum_{r, s=1}^{\infty}\left(A_{r s} \cdot\left(\frac{\mathrm{s} \pi}{\mathrm{b}}\right)+B_{r s} \cdot\left(\frac{\mathrm{r} \pi}{\mathrm{a}}\right)\right) \cdot \operatorname{Cos}\left(\frac{r \pi}{a}\right) x \cdot \operatorname{Cos}\left(\frac{s \pi}{b}\right) y \\
\gamma_{y z}=\psi_{y}+\partial w / \partial y=\sum_{r, s=1}^{\infty}\left\{\left(\frac{\mathrm{s} \pi}{\mathrm{b}}\right) \cdot W_{r s}+B_{r s}\right\} \cdot \operatorname{Sin}\left(\frac{r \pi}{a}\right) x \cdot \operatorname{Cos}\left(\frac{s \pi}{b}\right) y \\
\gamma_{x z}=\psi_{x}+\partial w / \partial x=\sum_{r, s=1}^{\infty}\left\{\left(\frac{\mathrm{r} \pi}{\mathrm{a}}\right) \cdot W_{r s}+A_{r s}\right\} \cdot \operatorname{Cos}\left(\frac{r \pi}{a}\right) x \cdot \operatorname{Sin}\left(\frac{s \pi}{b}\right) y
\end{gathered}
$$

In the above equations, $z$ is thickness coordinate of the structure. Using Equation (6), stressstrain relations for any lamina in the laminated plate with respect to $x, y$ and $z$ axes are given by:

$$
\left[\begin{array}{l}
\sigma_{x x} \\
\sigma_{y y} \\
\sigma_{x y}
\end{array}\right]=\left[\begin{array}{lll}
\bar{Q}_{11} & \bar{Q}_{12} & \bar{Q}_{16} \\
\bar{Q}_{12} & \bar{Q}_{22} & \bar{Q}_{26} \\
\bar{Q}_{16} & \bar{Q}_{26} & \bar{Q}_{66}
\end{array}\right]_{k}\left[\begin{array}{l}
\varepsilon_{x} \\
\varepsilon_{y} \\
\gamma_{x y}
\end{array}\right] ;\left[\begin{array}{l}
\sigma_{x z} \\
\sigma_{y z}
\end{array}\right]=\left[\begin{array}{ll}
\bar{Q}_{55} & \bar{Q}_{54} \\
\bar{Q}_{54} & \bar{Q}_{44}
\end{array}\right]_{k}\left[\begin{array}{l}
\gamma_{x z} \\
\gamma_{y z}
\end{array}\right]
$$

\section{RESULTS AND DISCUSSION}

In order to validate the present method and demonstrate its capability in predicting the free vibration response of composite plates with and without SMA wires, the results obtained from the present method were compared with those determined in Ostachowicz (1999) and Khalili et al. (2005). 


\subsection{Verifying of the Present Results}

First, a ten-layered carbon/epoxy plate with size of $20 \mathrm{~cm} \times 20 \mathrm{~cm}$ and each layer's thickness of $\mathrm{h}=0.269 \mathrm{~mm}$ was analyzed. The material properties were as follows:

$$
\begin{array}{ll}
\mathrm{E}_{11}=120 \mathrm{GPa} & v_{12}=v_{23}=0.33 \\
\mathrm{E}_{22}=\mathrm{E}_{33}=7.9 \mathrm{GPa} & \rho=1580 \mathrm{~kg} / \mathrm{m}^{3} \\
\mathrm{G}_{12}=\mathrm{G}_{13}=\mathrm{G}_{23}=5.5 \mathrm{GPa} &
\end{array}
$$

Stacking sequence of laminate was: $[0 / \theta / 0 /-\theta / 0]_{s}$. The role of fibers' orientation of laminas in the plate was obtained considering a sequence $0 / \theta / 0 /-\theta \cdots$ of the fiber' orientations with respect to $x$ axis.

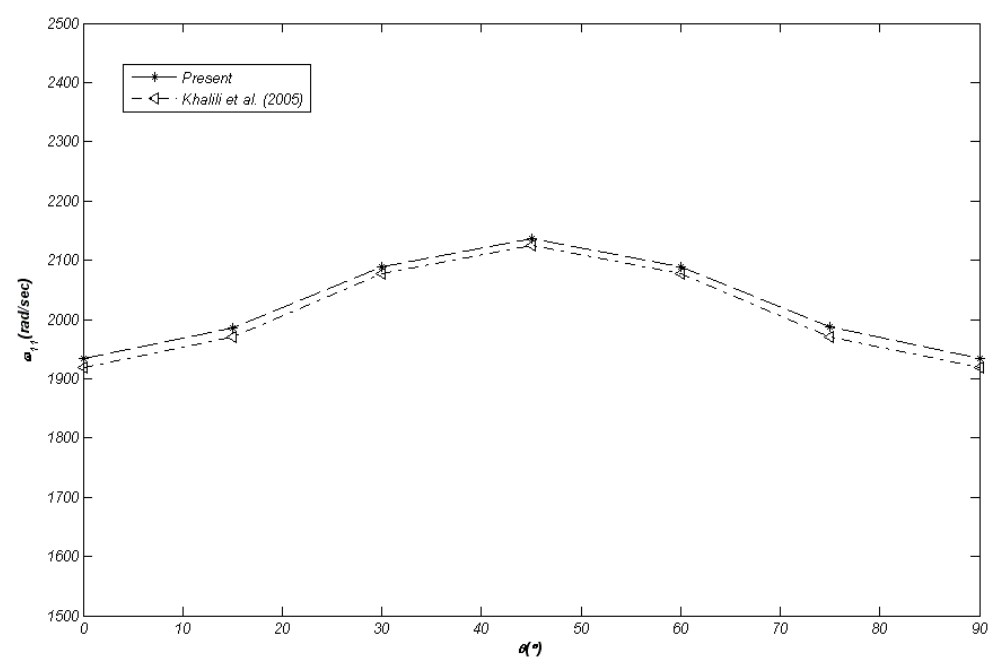

Figure 3 Comparison of the variation of natural frequency versus different fiber orientation angles by Khalili et al. (2005).

First, the dynamic analysis was carried out to evaluate natural frequencies of vibrations of the plate without SMA wires, using the present method as well as the method in Khalili et al. (2005). The results are compared in Figure 3. It is observed that the maximum difference between the frequencies obtained by two methods was less than $2 \%$. Because the effect of temperature on the composite medium and the SMA wires were considered in the present results, there was small discrepancy in the results. Second, the dynamic analysis was carried out to evaluate natural frequencies of vibrations of the plate embedded SMA wires, using the present method as well as the FEM in Ostachowicz (1999). Mechanical and physical properties of the shape memory alloys strongly depend on temperature and initial stresses (Ostachowicz, 1999).

Changes in temperature and initial stresses involve changes in the volume fraction of martensite in the alloys. During the martensite transformation, recovery stresses appear. These recovery stresses are not only a function of the temperature of the alloys but also depend on initial strains. In Fig- 
ure 1, the SMA wires recovery stresses versus the temperature for four different initial strains are presented (Ostachowicz, 1999). It can be easily noticed that increase in the initial strains involved higher recovery stresses in the SMA wires and simultaneous changes in the temperatures of phase transformation were observed.

In this study, only a uniform temperature distribution in the plate was considered. The following material properties for the SMA wires were also used: austenite start temperature $T_{s}=37.8^{\circ} \mathrm{C}$ and austenite finish temperature $T_{f}=62.8^{\circ} \mathrm{C}$. As an example, a simply supported square graphite-epoxy plate was investigated. The eight layer plate had the following orientation of graphite fibers $[0 / 90 / 90 / 0]_{S}$. Dimensions and material properties of the plate and SMA wires are given in Table 1.

Table 1 Geometrical and material properties of simply supported square graphite-epoxy plate.

\begin{tabular}{lllll}
\hline \hline $\mathrm{E}_{\mathrm{f}}=275.6$ & $\mathrm{GPa}$ & $\mathrm{E}_{\mathrm{m}}=3.43 \mathrm{GPa}$ & $\mathrm{E}_{\mathrm{s}}=70.1 \mathrm{GPa}$ \\
$\mathrm{G}_{\mathrm{f}}=114.8 \mathrm{GPa}$ & $\mathrm{G}_{\mathrm{m}}=1.27 \mathrm{GPa}$ & $\mathrm{G}_{\mathrm{s}}=24.86 \quad \mathrm{GPa}$ \\
$v_{\mathrm{f}}=0.2$ & & $\mathrm{v}_{\mathrm{m}}=0.35$ & $\mathrm{v}_{\mathrm{s}}=0.3$ \\
$\rho_{\mathrm{f}}=1900 \mathrm{Kg} / \mathrm{m}^{3}$ & $\rho_{\mathrm{m}}=1250 \mathrm{Kg} / \mathrm{m}^{3}$ & $\rho_{\mathrm{s}}=6450 \quad \mathrm{Kg} / \mathrm{m}^{3}$ \\
$\alpha_{\mathrm{f}}=24.4$ & $1 /{ }^{\circ} \mathrm{C}$ & $\alpha_{\mathrm{m}}=64.8 \quad 1 /{ }^{\circ} \mathrm{C}$ & $\mathrm{v}_{\mathrm{s}}=5 \%$ \\
$\mathrm{v}_{\mathrm{f}}=20 \%$ & $\mathrm{a}=\mathrm{b}=0.6 \mathrm{~m}$ & $\mathrm{~h}=0.008 \mathrm{~m}$ \\
& & & $\mathrm{~T}_{\mathrm{s}}^{\mathrm{SA}}=37.8^{\circ} \mathrm{C}$ \\
& & & $\mathrm{T}_{\mathrm{f}}^{\mathrm{SA}}=62.8^{\circ} \mathrm{C}$ \\
\hline \hline
\end{tabular}

The properties of graphite fibers are shown by index (f) and properties of epoxy matrix and SMA wires are shown by indices $(\mathrm{m})$ and $(\mathrm{s})$, respectively. The plate was modeled with 64 plate elements and the mesh size was 8 X 8 in (Ostachowicz, 1999). The volume fraction of graphite fibers in each layer was 0.2. In the present example, the SMA wires covered only $5 \%$ of the cross-sectional area of the plate. They were embedded in the neutral plane of the plate. It should be noticed that the relationship between the area and the internal forces was linear.

Certainly, the relationship between changes in natural frequencies and internal forces was not linear. In this paper, $\boldsymbol{\omega}$ and $\overline{\boldsymbol{\omega}}$ are natural frequencies of plates with and without SMA wires, respectively. Also, $N F=\omega / \bar{\omega}$ is the dimensionless frequency parameter. All results of the presented numerical calculations were related to those obtained for the same plate without the SMA wires at room temperature of $20^{\circ} \mathrm{C}$. Comparison of the present results of the variations of dimensionless natural frequency parameter $\omega / \bar{\omega}$ versus different temperatures of the SMA wires with results of Ostachowicz (1999) is shown in Table 2.

In addition, variation of the second and third dimensionless natural frequency parameters $\omega / \bar{\omega}$ versus different temperatures of the SMA wires obtained from the present method are presented in the table. The agreement between the results was quite good. The results of numerical investigations illustrated the influence of temperature of the SMA wires upon changes in bending natural frequencies of the plate. It can be clearly observed that the activation process of the SMA wires 
involved increase in the bending natural frequencies. This effect incaresed when both temperature and tensile pre-strains were higher.

The results obtained in this work demonstrated potential effectiveness of SMA fiberreinforcement in composite structural elements in the control of vibrations. However, it must be noticed that the speed of response of changes in natural frequencies using the SMA wires was low. For this reason, SMA actuators can be used when high frequency controlling is not required. Nevertheless, internal forces generated in the SMA wires were much higher than those in other classes of smart materials, which allows for their application in these engineering applications.

Figures 4(a) and 4(b) show the first nine three dimensional transverse mode shapes and strains of the bottom layer of hybrid composite plate. The SMA temperature was constant and equal to austenite finish temperature $T_{f}=62.8^{\circ} \mathrm{C}$. These figures demonstrate that the lowest natural frequency was $\omega_{11}=669.321 \mathrm{rad} / \mathrm{sec}$.

Table 2 Comparison of the variation of fundamental dimensionless natural frequency parameters $\omega / \bar{\omega}$ vs. different temperatures of the SMA wires with those in Ostachowicz et al. (1999).

\begin{tabular}{cccccc}
\hline \hline \multicolumn{2}{c}{ Ostachowicz et al. $(\mathbf{1 9 9 9})$} & \multicolumn{3}{c}{ Present } \\
\hline$\sigma_{r}(M p a)$ & $T\left({ }^{\circ} \mathrm{C}\right)$ & $\mathbf{4 8 3 . 5} \varpi_{11}=$ & $\mathbf{4 8 6 . 6} \varpi_{11}=$ & $1264.8 \varpi_{12}=$ & $\varpi_{22}=1944$ \\
\hline $4 \% \mathcal{E}_{L}=$ & $S M A$ & $\omega_{11} / \varpi_{11}$ & $\omega_{11} / \varpi_{11}$ & $\omega_{12} / \varpi_{12}$ & $\omega_{22} / \varpi_{22}$ \\
\hline 9.2658 & 35.08 & $\mathbf{1 . 0 0 0 0}$ & $\mathbf{1 . 0 0 3 2}$ & 0.9871 & 0.9891 \\
16.2963 & 38.45 & $\mathbf{1 . 0 3 7 8}$ & $\mathbf{0 . 9 9 6 4}$ & 0.9814 & 0.9874 \\
26.9627 & 43.62 & $\mathbf{1 . 1 0 5 9}$ & $\mathbf{1 . 0 0 9 1}$ & 0.9814 & 0.9906 \\
78.1572 & 50.52 & $\mathbf{1 . 1 8 9 4}$ & $\mathbf{1 . 0 9 8 4}$ & 0.9933 & 1.0141 \\
128.1107 & 56.44 & $\mathbf{1 . 2 9 4 5}$ & $\mathbf{1 . 1 7 8 7}$ & 1.0047 & 1.0364 \\
201.2183 & 65.46 & $\mathbf{1 . 2 8 9 4}$ & $\mathbf{1 . 2 8 9 7}$ & 1.0222 & 1.0690 \\
221.2023 & 75.33 & $\mathbf{1 . 3 1 9 7}$ & $\mathbf{1 . 3 1 1 1}$ & 1.0236 & 1.0755 \\
237.7668 & 85.33 & $\mathbf{1 . 3 3 6 3}$ & $\mathbf{1 . 3 2 6 4}$ & 1.0236 & 1.0802 \\
251.0479 & 95.33 & $\mathbf{1 . 3 6 5 5}$ & $\mathbf{1 . 3 3 6 7}$ & 1.0227 & 1.0834 \\
264.2970 & 105.25 & $\mathbf{1 . 3 5 3 0}$ & $\mathbf{1 . 3 4 6 9}$ & 1.0219 & 1.0866 \\
272.3818 & 115.22 & $\mathbf{1 . 3 7 3 9}$ & $\mathbf{1 . 3 4 9 0}$ & 1.0194 & 1.0872 \\
284.4064 & 125.24 & $\mathbf{1 . 3 6 3 0}$ & $\mathbf{1 . 3 5 6 9}$ & 1.0180 & 1.0897 \\
292.4773 & 135.33 & $\mathbf{1 . 3 6 5 4}$ & $\mathbf{1 . 3 5 9 3}$ & 1.0157 & 1.0904 \\
299.0952 & 145.46 & $\mathbf{1 . 3 6 6 5}$ & $\mathbf{1 . 3 5 9 1}$ & 1.0127 & 1.0904 \\
302.7302 & 155.44 & $\mathbf{1 . 3 5 9 7}$ & $\mathbf{1 . 3 5 7 3}$ & 1.0102 & 1.0898 \\
304.9689 & 162.08 & $\mathbf{1 . 3 5 7 8}$ & $\mathbf{1 . 3 5 1 8}$ & 1.0065 & 1.0881 \\
307.0120 & 165.30 & $\mathbf{1 . 3 5 6 9}$ & $\mathbf{1 . 3 4 7 3}$ & 1.0034 & 1.0867 \\
\hline \hline
\end{tabular}




\subsection{Parametric Studies of the Free Vibration Problem}

The effect of some of the geometrical, physical and material parameters on dynamic response of the hybrid composite plates was studied in this section. These parameters were some of the important factors affecting dynamic response and design of the smart structures. It was established that the dynamic behavior of the smart hybrid composite plate depended on various parameters such as volume fraction, temperature and tensile pre-strain of the SMA wires and the aspect ratio and fiber orientation of the laminated hybrid plate. Some of numerical results (which have not been reported in the literature thus far) were presented in this section. The used hybrid composite plate was symmetric. In all of the parametric studies, other than studying temperature effect, the SMA temperature was constant and equal to austenite finish temperature $\mathrm{T}_{\mathrm{f}}=62.8^{\circ} \mathrm{C}$.

The plate boundary conditions were simply supported. The plate consisted of eight layers of composite material with the angle of graphite fibers $[0 / 90 / 90 / 0]_{\mathrm{S}}$. The dimensions and material properties of the plate and SMA wires are presented in Table 1. In order to study influence of stacking sequence of laminas, angle of plies of the composite plate was assumed to be $[S M A(0) / \theta /-\theta / 0]_{s}$ and the effect of $D_{16}$ and $D_{26}$ was negligible. In all of parametric studies other than studying pre-strain effect, the initial strain was assumed $\varepsilon_{L}=4 \%$.
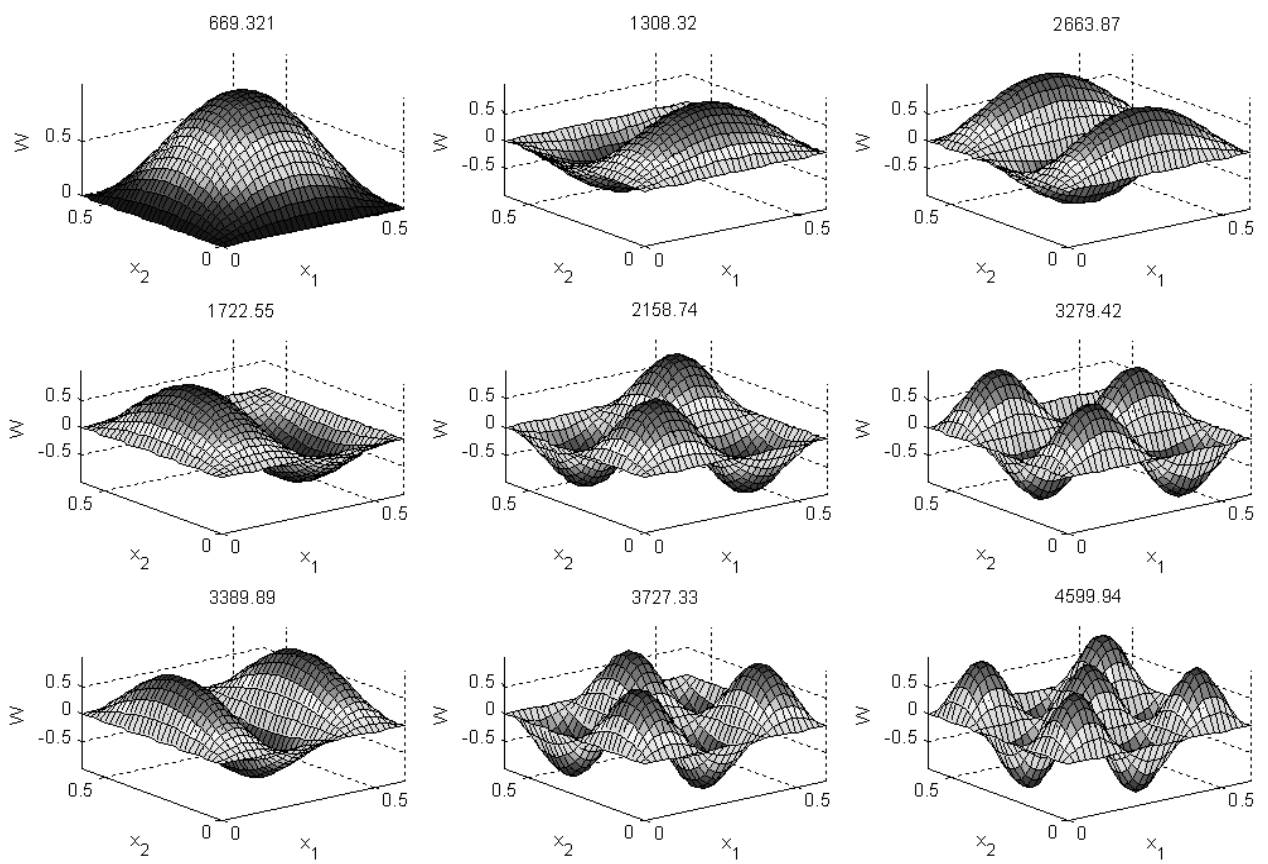

(a)

Figure 4 (a) The first nine three-dimensional transverse mode shapes, (b) the first nine three dimensional transverse modal strains of $t$ he smart hybrid composite plate. $x_{1}$ and $x_{2}$ show $\mathrm{x}$ and $\mathrm{y}$ axes. 

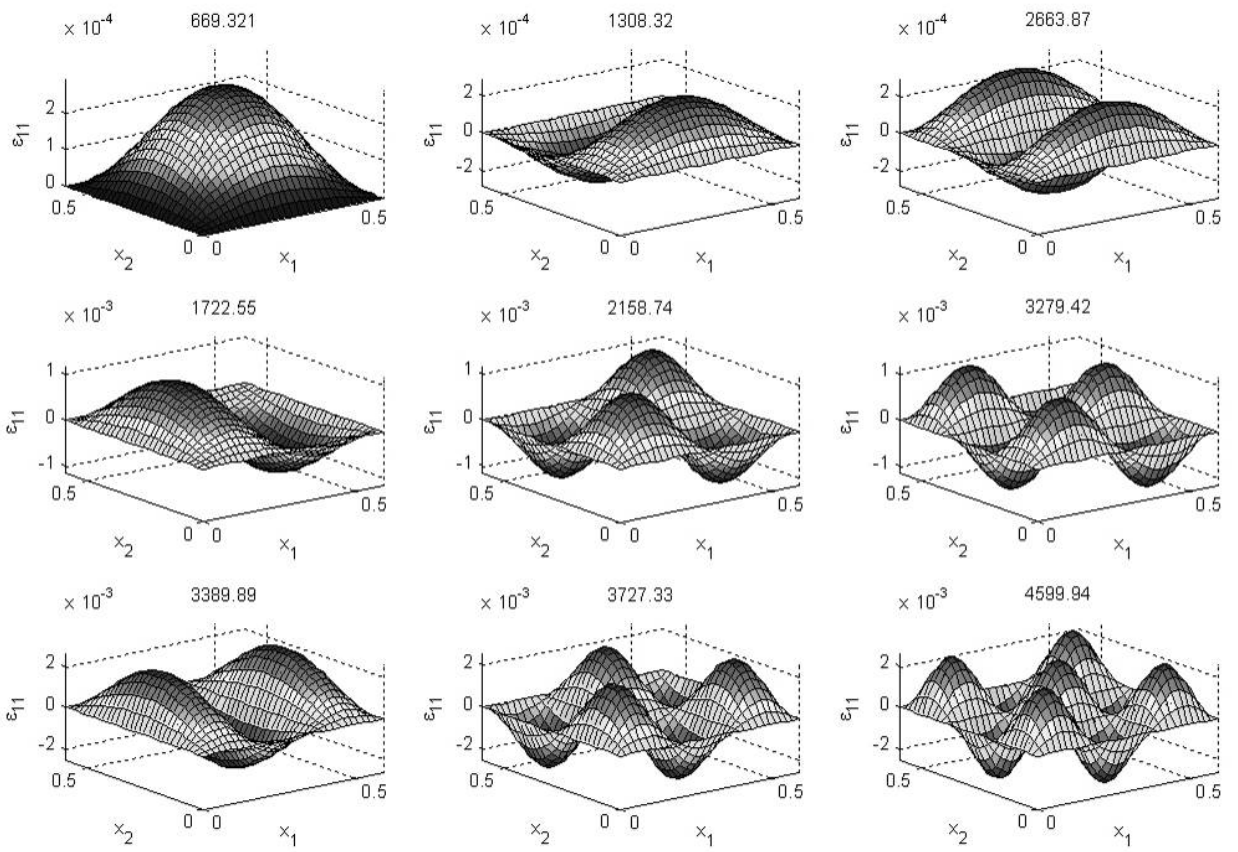

(b)

Figure 4 (continued) (a) The first nine three-dimensional transverse mode shapes, (b) the first nine three dimensional transverse modal strains of the smart hybrid composite plate. $x_{1}$ and $x_{2}$ show $\mathrm{x}$ and $\mathrm{y}$ axes.

\subsubsection{Effect of SMA Temperature on Natural Frequencies}

Effect of temperature of the SMA wires on dimensionless natural frequency parameter (NF) is shown in Figure 5. In this figure, relative changes in the first to the fifth bending natural frequencies are presented. The results of numerical investigations illustrated influence of the SMA fibers' temperature upon changes in bending natural frequencies of the analyzed plate. Figure 5 shows that effect of increasing SMA temperature on dimensionless fundamental NF was at the highest level.

The figure also shows that the variation of NF at temperatures between $T_{s}<T<T_{f}$ (austenite transformation range) was larger than the variation of NF at other temperatures. It can be clearly observed that the activation process of the SMA wires involved increase in the bending natural frequencies. This effect increased when both temperature and initial strains were higher. 


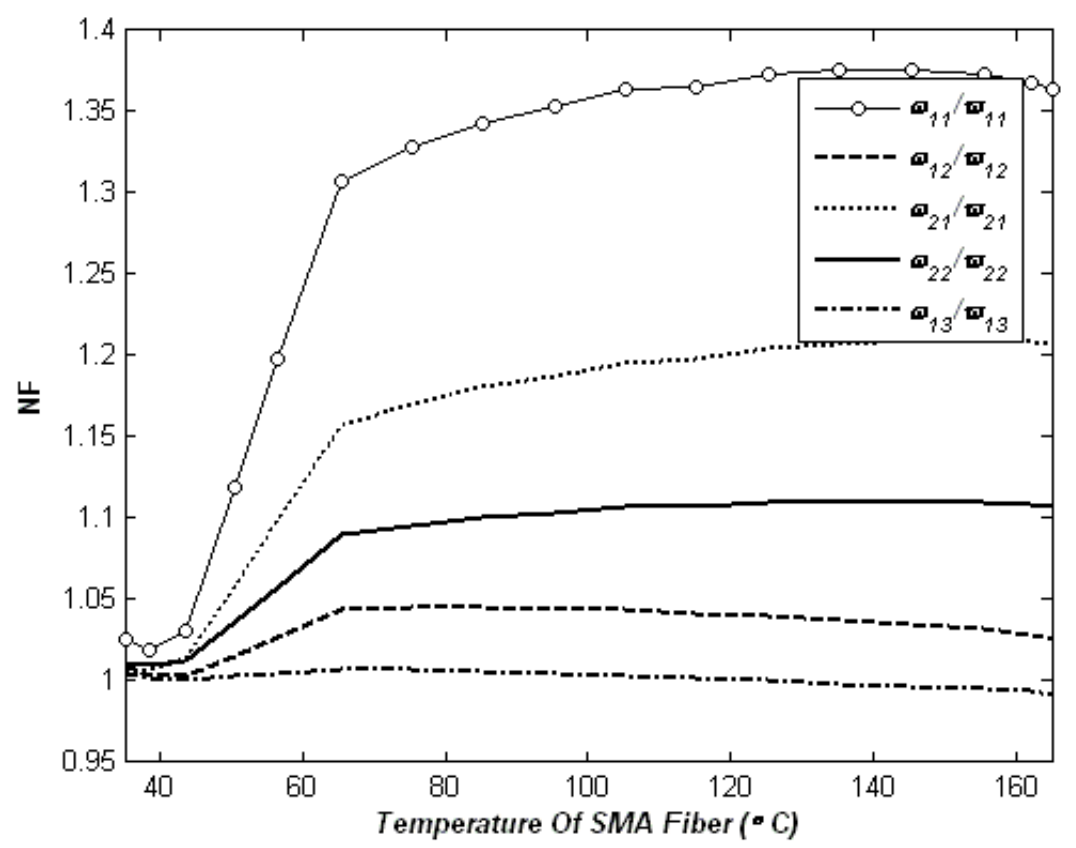

Figure 5 Effect of temperature of the SMA wires on dimensionless natural frequency parameter (NF).

\subsubsection{Effect of Tensile Pre-strain and Temperature of the SMA Wires on Free Vibration Re- sponse of Composite Hybrid Plate}

Influence of tensile pre-strain of the SMA wires (at the final temperature of austenite transformation $\mathrm{T}=62.8^{\circ} \mathrm{C}$ ) upon the relative changes of the first nine natural frequencies is shown in Figure 6. It can be clearly observed that the initial strain of SMA wires involved increase in the bending natural frequencies. Effect of both temperature and initial tensile pre-strain of the SMA wires on dimensionless natural frequency parameter $(\mathrm{NF})$ is shown in Figure 7 . This figure shows that the variation of NF at temperatures between $T_{s}<T<T_{f}$ (austenite transformation range) was larger than the variation of NF at other temperatures. It can be clearly observed that the activation process of the SMA wires involved increase in the bending natural frequencies. This effect was intensified when both temperature and initial strains were higher.

As shown in Figure 7, with thermal expanding of the composite graphite epoxy plate and the austenite phase of SMA wires $(\alpha \Delta T)$, the fundamental frequency of the plate considerably decreased as the SMA temperature increased (for $\varepsilon_{L}=1 \%$ to $\varepsilon_{L}<3 \%$ and $T>T_{f}$ ). This reduction of the fundamental frequency could be considerably compensated for by increasing tensile pre-strain of the SMA wires $\left(\varepsilon_{L}>3 \%\right)$. 


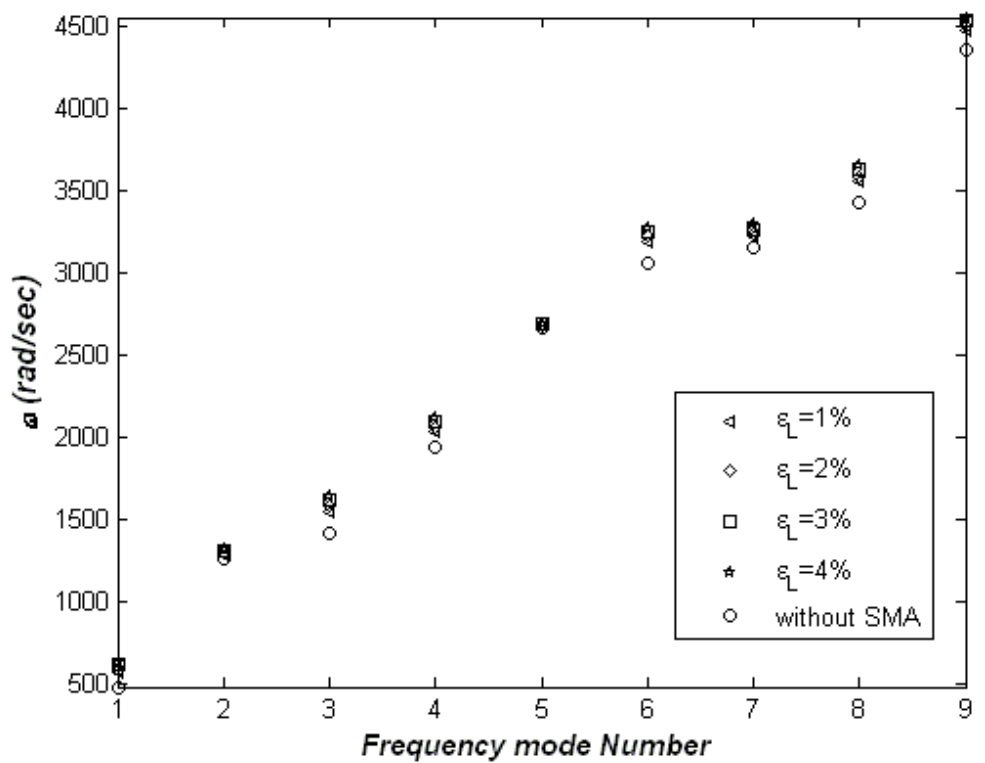

Figure 6 Influence of tensile pre-strain of the SMA wires upon the relative change of the first nine natural frequencies.

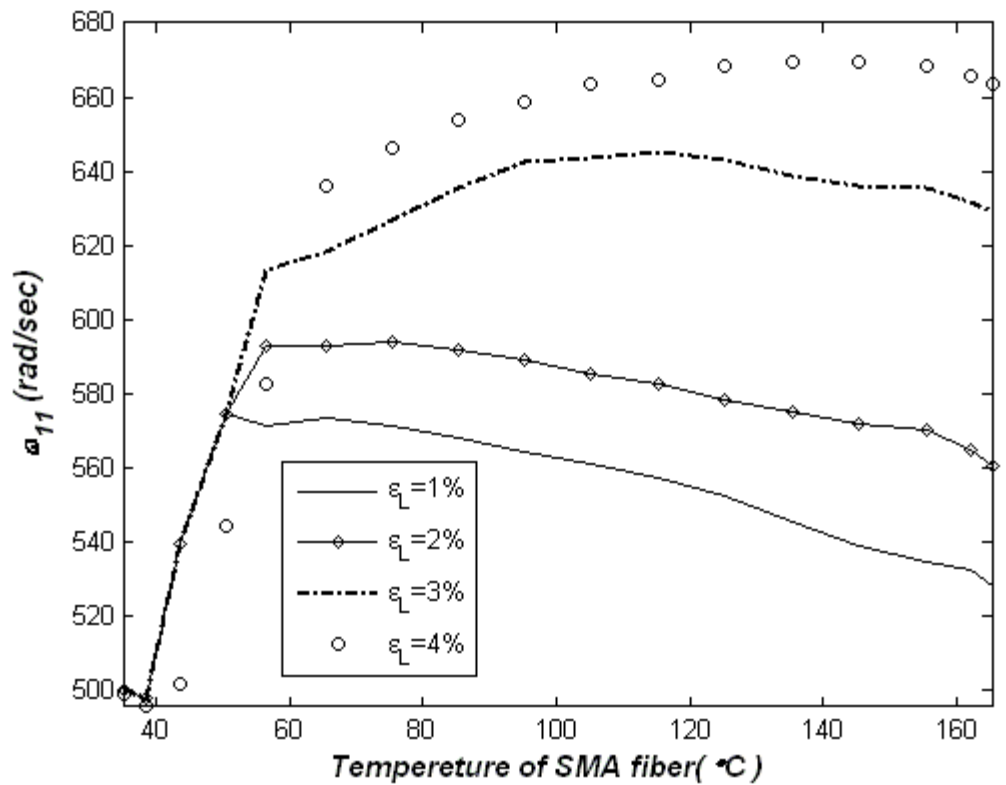

Figure 7 Influence of temperature and tensile pre-strain of SMA wires upon the relative change of the first natural frequency. 


\subsubsection{Effect of Tensile Pre-strain of the SMA Wires and the Plate Aspect Ratio on Fundamental Natural Frequency of the Plate}

Figure 8 shows that fundamental natural frequency increased with the increased aspect ratio $a / b$. Because, as aspect ratio $(a / b)$ increased, length of plate increased and the plate was closer to a beam. However, boundary conditions were applicable on all four edges, which resulted in the decrease of vertical and shear deformations and increase of stiffness of the plate as well as coupling effects between the membrane, bending and shear terms. Therefore, the bending stiffness and fundamental natural frequency increased with the increased aspect ratio $a / b$.

Effect of both aspect ratio of the composite plate and tensile pre-strain of the SMA wires on the first natural frequency is shown in Figure 8. It can be clearly observed that the activation process of the SMA wires involved increase in the bending natural frequencies only for $a / b<2.4$. The SMA wires were embedded along the $\mathrm{x}$ axis (along length of the plate). As the volume fraction of SMA wires decreased with the increase of the plate's aspect ratio $a / b$, the effect of tensile pre-strain and activation process of the SMA wires on increase of the first natural frequency was slight and negligible, especially for $a / b>2.4$. Since the increase of the aspect ratio represented decreased wire volume fraction in the composite plates, therefore, the recovery force acted into the plate became insignificant.

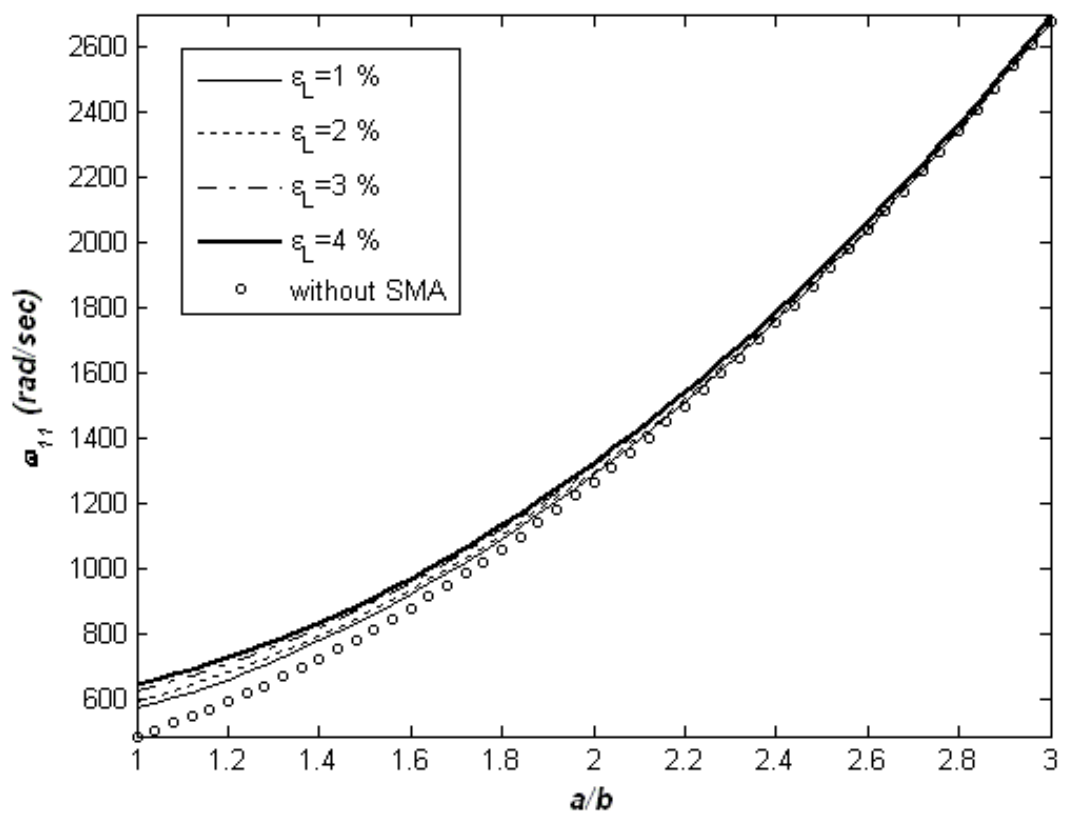

Figure 8 Influence of tensile pre-strain of SMA wires and aspect ratio of plate upon the relative change of the first natural frequency. 


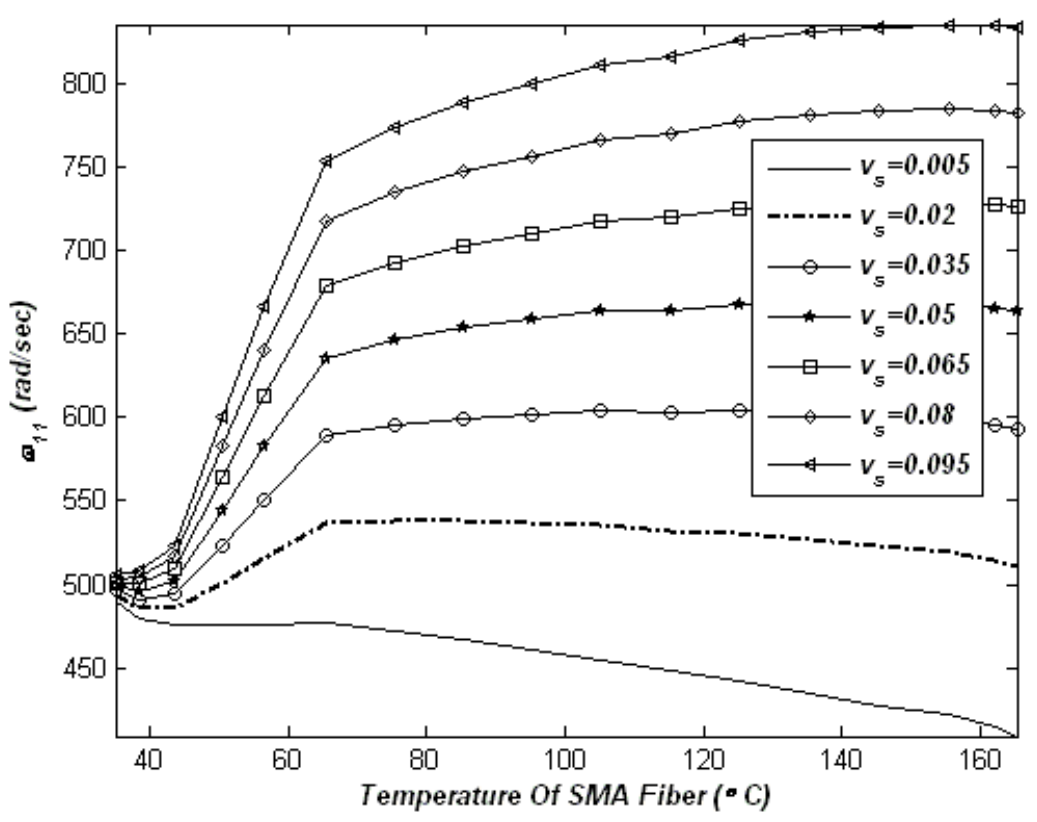

Figure 9 Effect of increasing volume fraction of SMA wires on fundamental natural frequency.

\subsubsection{Effect of Volume Fraction and Temperature of SMA Wires on Fundamental Natural Fre- quency of the Plate}

Effect of temperature and volume fraction of SMA wires on fundamental natural frequency is illustrated in Figure 9. The results of numerical investigations illustrated the influence of the SMA volume fraction upon changes in bending natural frequencies of the analyzed plate. The figure shows that the variation of fundamental natural frequency at temperatures between $T_{s}<T<T_{f}$ (austenite transformation range) was larger than the variation of fundamental natural frequency at other temperatures. The volume fractions of the SMA wires were considered 0.005 to 0.095 . The wires were placed only along the $\mathrm{x}$ direction. It is evident in Figure 9 that, by increasing volume fraction of the SMA wires, the fundamental natural frequency increased. As shown in the figure, with thermal expansion of composite graphite epoxy plate and austenite phase of the SMA wires $(\alpha \Delta T)$, the fundamental frequency of the plate decreased considerably as the SMA temperature increased (for $V_{s}=0.005$ to $V_{s}=0.035$ and $T>T_{f}$ ). This reduction of the fundamental frequency could be considerably compensated for by increasing volume fraction of the SMA wires $\left(V_{s}>0.035\right)$.

\section{CONCLUSIONS}

In this research, free vibration response of smart hybrid composite structures was studied using the simultaneous application of FSDT and Fourier series to analytically solve system of the governing differential equations of the plate. The results of the present research demonstrated that: 
1) Using of the SMA wires inside the hybrid composite plates changed natural frequencies as well as global behavior of the structure.

2) Some of the geometrical, physical and material parameters like volume fraction and tensile pre-strain of the SMA wires and the plate's aspect ratio were important factors affecting free vibrations response and design of the structures.

3) The variation of dimensionless natural frequency parameter (NF) at temperatures between $T_{s}<T<T_{f}$ (austenite transformation range) was larger than the variation of NF at other temperatures.

4) Activation process of the SMA wires involved increase in the bending natural frequencies. This effect was intensified when both temperature and initial strains were higher.

5) Tensile pre-strain of the SMA wires involved increase in the bending natural frequencies.

6) The bending stiffness and fundamental natural frequency increased with the increase in the plate's aspect ratio $a / b$.

7) Activation process of the SMA wires involved increase in the bending natural frequencies only for $a / b<2.4$.

8) As the volume fraction of SMA wires decreased with the increase of the plate's aspect ratio $a / b$, effect of tensile pre-strain and activation process of the SMA wires on increase of the first natural frequency was slight and ignorable, especially for $a / b>2.4$.

9) By increasing volume fraction of the SMA wires, the fundamental natural frequency increased.

10) With thermal expansion of the composite graphite epoxy plate and the austenite phase of SMA wires $(\alpha \Delta T)$, the fundamental frequency of the plate decreased considerably as the SMA temperature increased (For $V_{s}=0.005$ to $V_{s}=0.035$ and $T>T_{f}$ ). This reduction of the fundamental frequency could be considerably compensated for by increasing volume fraction of the SMA wires $\left(V_{s}>0.035\right)$.

\section{References}

Altenbach, H., Altenbach, J. and Kissing, W., (2004). Mechanics of composite structural elements, Springer, 122-297.

Birman, V., Chandrashekhara, K. and Sain., S., (1996). An Approach to Optimization of Shape Memory Alloy Hybrid Composite Plates Subjected to Low-velocity Impact, Composites: Part B, 27B: 439-446.

Khalili, M. R., Malekzadeh, K. and Mittal, R. K., (2005). A new approach to static and dynamic analysis of composite plates with different boundary conditions, Composite Structures, 69: 149-155.

Khalili , S. M. R., Shokuhfar, A. and Ashenai Ghasemi, F., (2007). Effect of smart stiffening procedure on lowvelocity impact response of smart structures, Journal of Materials Processing Technology, 190: 142-152.

Lau, K. T. (2002). Vibration characteristics of SMA composite beams with different boundary conditions, Materials and Design. 23: 741-749.

Mindlin, R. D. (1951). Influence of rotary inertia and shear on flexural motions of isotropic elastic plates, J. of Appl. Mech., 18: 31-38. 
Ni, Qing-Q., Zhang, Run-xin., Natsuki, T. and Iwamoto, M., (2007). Stiffness and vibration characteristics of SMA/ER3 composites with shape memory alloy short fibers, Composite Structures, 79: 501-507.

Ostachowicz, W. M., Krawczuk, A. Zak., (1999). Natural frequencies of a multilayer composite plate with shape memory alloy wires, Finite Elements in Analysis and Design, 32: 71-83.

Ostachowicz, W. M., Krawczuk, A. Zak., (2000). Dynamics and buckling of a multilayer composite plate with embedded SMA wires, Composite Structures, 48: 163-167.

Ostachowicz, W. M., Kaczmarczyk, S., (2001). Vibration of composite plate with SMA fibers in a gas stream with defect of the type of delamination, Composite Structures, 54: 305-311.

Park, J. S., Kim, J. H., Moon, S. H., (2004). Vibration of thermally post-buckled composite plates embedded with shape memory alloy fibers, Composite Structures, 63: 179-188.

Reddy, J. N. (1997). Introduction to composite materials, Mechanics of laminated composite plates and shells, Second Edition, 88, CRC Press, United States of America.

Whitney, J. M. and Pagano N. J., (1970). Shear deformation in heterogeneous anisotropis plates, J. Appl. Mech., 37: 1031-1036.

Wilkinson, J. H. (1992). The algebraic eigenvalue problem, Clarendon Press. Oxford. in : S.S. Rao., Mechanical Vibrations. Pergamon Press. Oxford, 285-300.

Zhang, Run-xin, Ni, Qing-Q., Masuda, A., Yamamura, T. and Iwamoto, M., (2006). Vibration characteristics of Laminated Composite Plates with embedded Shape memory alloys, Composite Structures, 74(4): 389-398. 